\title{
Selenium-binding protein 1 is down-regulated in malignant melanoma
}

\author{
Mandy Schott ${ }^{1, *}$, Miriam M. de Jel ${ }^{1, *}$, Julia C. Engelmann², Philipp Renner ${ }^{3}$, Edward \\ K. Geissler ${ }^{3}$, Anja K. Bosserhoff ${ }^{1}$ and Silke Kuphal ${ }^{1}$ \\ ${ }^{1}$ University of Erlangen, Institute of Biochemistry, Biochemistry and Molecular Medicine, Erlangen, Germany \\ ${ }^{2}$ University of Regensburg, Institute of Functional Genomics, Statistical Bioinformatics, Regensburg, Germany \\ ${ }^{3}$ Department of Surgery, University Medical Center Regensburg, Regensburg, Germany \\ *Co-first authors
}

Correspondence to: Silke Kuphal, email: Silke.Kuphal@fau.de

Keywords: malignant melanoma; selenium-binding protein 1 (SELENBP1); glutathione peroxidase 1 (GPX1); Grm 1 mouse model Received: March 22, $2017 \quad$ Accepted: December 28, $2017 \quad$ Published: January 02, 2018

Copyright: Schott et al. This is an open-access article distributed under the terms of the Creative Commons Attribution License 3.0 (CC BY 3.0), which permits unrestricted use, distribution, and reproduction in any medium, provided the original author and source are credited.

\section{ABSTRACT}

Selenium-binding protein 1 (SELENBP1) expression is reduced in various epithelial cancer entities compared to corresponding normal tissue and has already been described as a tumor suppressor involved in the regulation of cell proliferation, senescence, migration and apoptosis. We identified SELENBP1 to be down-regulated in cutaneous melanoma, a malignant cancer of pigment-producing melanocytes in the skin, which leads to the assumption that SELENBP1 also functions as tumor suppressor in the skin, as shown by others e.g. for prostate or lung carcinoma.

However, in vitro analyses indicate that SELENBP1 re-expression in human melanoma cell lines has no impact on cell proliferation, migration or tube formation of the tumor cells themselves when compared to control-transfected cells. Interestingly, supernatant taken from melanoma cell lines transfected with a SELENBP1 reexpression plasmid led to suppression of vessel formation of HMEC cells. Furthermore, SELENBP1 re-expression alters the sensitivity of melanoma cells for Vemurafenib treatment.

The data also hint to a functional interaction of SELENBP1 with GPX1 (Glutathione peroxidase 1). Low SELENBP1 mRNA levels correlate inversely with GPX1 expression in melanoma. The re-expression of SELENBP1 combined with down-regulation of GPX1 expression led to reduction of the proliferation of melanoma cells. In summary, SELENBP1 influences the tumor microenvironment and SELENBP1 action is functionally influenced by GPX1.

\section{INTRODUCTION}

Malignant melanoma is the most aggressive form of skin cancer, and its incidence is rising at alarming rates [1]. To study melanoma development and progression in vivo the transgenic mouse strain $\operatorname{Tg}(\mathrm{Grml})$ was generated [2]. $\operatorname{Tg}(\mathrm{Grm} /)$ mice spontaneously develop pigmented lesions within a short time of latency and with $100 \%$ penetrance due to the melanocyte-specific expression of a metabotropic glutamate receptor 1 (Grml) transgene. As the $\mathrm{Grm} 1$ transgene is placed under control of the melanocyte specific Dct promoter, Grm 1 is specifically overexpressed in cells of melanocytic origin leading to both cutaneous and uveal melanoma [2,3]. Interestingly, GRM1 up-regulation is also present in human melanoma cell lines and tissues $[2,4,5]$. Since transcriptome sequencing did not reveal melanoma-associated mutations or single nucleotide variations in $\operatorname{Tg}(\mathrm{Grm} /)$ mice [6], we assume, besides Grm1 overexpression, epigenetic events or changes in gene expression are important for driving 
melanoma development and progression in this mouse model.

Selenium is a micronutrient for a number of physiological biological processes in the human body. Selenium supplementation at nutritional dosage (nM range) has been extensively studied for its preventive effects against various cancers [7-9], implicating that selenium-containing proteins are likely to play crucial roles in selenium-mediated cancer prevention. Selenium-binding protein 1 (SELENBP1, $S B P 1, h S P 56$ ), a member of the selenoprotein family, has been shown to mediate the intracellular transport of selenium [10]. SELENBP1 is expressed in a wide range of normal human tissues, but is suppressed in diverse types of epithelial cancers such as prostate, stomach, colon, lung, thyroid and ovary [11-14]. SELENBP1 down-regulation is associated with tumor progression as well as poor clinical outcome [15-18]. Moreover, several studies show that SELENBP1 is involved in the regulation of cellular processes including proliferation, migration, senescence and apoptosis [16, 18-20].

Glutathione peroxidase 1 (GPX1) is also an important selenium-containing protein which is ubiquitously expressed. GPX1 is an antioxidant-enzyme that scavenges organic hydroperoxides using reducing equivalents from glutathione and protects cells from reactive oxygen species (ROS) [21-23]. Among the 25 human selenocysteinecontaining proteins, there is considerable evidence that the cytosolic form of GPX1 is associated with cancer risk. Given the cumulative data indicating possible roles of both SELENBP1 and GPX1 in cancer development and/or outcome, the interaction of these two selenium-associated proteins was investigated in several model systems [24].

In the present study we demonstrate that SELENBP1 is not only down-regulated in malignant melanoma samples of a murine $\operatorname{Tg}(\mathrm{Grm} 1)$ model for spontaneous melanoma but also in human melanoma cell lines and tissues, primarily arguing that SELENBP1 may be an important tumor suppressor in malignant melanoma. Interestingly, no direct influence of SELENBP1 reexpression on melanoma cells themselves was observed. However, SELENBP1 re-expression changes tube formation capacity of HMEC cells thus having an effect on the tumor microenvironment in melanoma.

\section{RESULTS}

\section{SELENBP1 is suppressed in murine melanoma tissues of the $\mathrm{Tg}(\mathrm{Grm} 1)$ mouse model}

To analyze differences in gene expression profiles between nevi and melanoma samples, a RNA-sequencing analysis was performed on two nevi and two melanoma samples from $\operatorname{Tg}(\mathrm{Grm} 1)$ mice. The sequence data is publicly available from NCBI, BioProject PRJNA237546. In this analysis SelenBP1 was found to be one of the strongest down-regulated gene of a total of 1,085 downregulated genes in $\operatorname{Tg}(\mathrm{Grm} 1)$ melanoma samples compared with nevi tissue. Whereas nevi samples reach a number of 3,674 reads on average, melanoma samples display a mean number of only 132 reads for the SelenBP1 gene (Figure 1A). The lack of SelenBP1 data regarding its role in melanoma prompted us to analyze its relevance in this kind of cancer. Studies with additional tissue samples confirmed SelenBP1 down-regulation in murine melanoma samples both on mRNA (Figure 1B) and protein level (Figure 1C). The murine nevi and tumor samples showed strong pigmentation; therefore, it was necessary to perform immunofluorescence instead of immunohistochemistry. Immunofluorescence analyses with murine nevi samples of the tail indicate the specificity of SelenBP1 staining (green) in pigmented melanocytes (Figure 1D), whereas melanomas are only positively stained for DAPI (localization of the nuclei). Hence, this in vivo result reveals down-regulation of SelenBP1 in murine melanoma samples.

\section{SELENBP1 is down-regulated in human melanoma cell lines and tissues}

To investigate whether data obtained from the murine melanoma mouse model are relevant to the human system, qRT-PCR analyses were performed with normal human epidermal melanocytes (NHEM) and human melanoma cell lines. Compared to NHEM, melanoma cells display a significant decrease in SELENBP1 mRNA expression (Figure 2A). SELENBP1 suppression in melanoma cells (PT, primary tumor; MET, metastasis) was confirmed on protein level showing reduced SELENBP1 protein amounts in western blot analysis compared to healthy NHEM (Figure 2B), and in immunofluorescence staining of two representative cell lines (Figure 2C).

Silencing of the SELENBP1 gene due to hypermethylation and chromatin remodeling appears to be frequently involved in tumorigenesis of colorectal cancer [25]. To further investigate the roles of $\mathrm{CpG}$ island methylation and histone deacetylation in transcriptional silencing of SELENBP1 in melanoma, we induced DNA demethylation and inhibited histone deacetylase. The cell lines were incubated with $5 \mu \mathrm{M}$ 5-Aza-deoxycytidine for $72 \mathrm{~h}$, followed by treatment with $300 \mathrm{nM}$ TSA for $4 \mathrm{~h}$. The treatment of the cells did not lead to an induction of SELENBP1 expression (Supplementary Figure 1A). These results suggest that epigenetic silencing by hypermethylation of the SELENBP1 promoter is not involved in its regulation in melanoma. Speculating that a SELENBP1 gene mutation could be responsible for the low expression levels, we studied the COSMIC (Catalogue of Somatic Mutations in Cancer)-home page $[26,27]$. Only 13 of 1,009 analyzed malignant melanoma samples in total harbor a SELENBP1 mutation (data not shown). The result was supported by the finding that 
RNA-sequencing analysis of the $\operatorname{Tg}(\mathrm{Grm} 1)$ mouse model uncovered also no melanoma-associated mutations or single nucleotide variations [6].

Next, we speculated that SELENBP1 can be regulated by hypoxia, a condition which is cumulative and endogenously found in melanomas. A previous publication of our own group revealed constitutively induction of HIF-1 $\alpha$ expression in melanoma cells [28]. Therefore, we investigated the hypoxic effects on SELENBP1 expression using Desferrioxamine (DFX) and 2, 2'-dipyridyl (DP) as iron chelators and inhibitors of prolyl hydroxylases (PHDs). Both chemical compounds mimic hypoxic effects and increase the SELENBP1 amount on mRNA level as exemplarily shown for the melanoma cell lines Sk-Mel-28 and Mel Juso (Supplementary Figure 1B). Hence, hypoxic effects induce SELENBP1 expression.

\section{SELENBP1 is down-regulated in human melanoma in vivo}

To analyze SELENBP1 expression in human in vivo samples in general, we first performed qRT-
PCR with mRNA samples from melanoma patients from isolated nevi tissue, isolated primary melanocytes and keratinocytes (Figure 3A). Nevi and melanocytes showed high expression levels of SELENBP1, while keratinocytes harbor lower levels of SELENBP1 mRNA. In addition, SELENBP1 mRNA expression was analyzed in human tissue samples from melanoma patients, displaying a decrease in SELENBP1 mRNA compared to normal skin (NS) (Figure 3B). Evaluating geoprofile data (GDS1375) from melanoma patients confirmed the previous results that normal skin samples (NS) express high amounts of SELENBP1 mRNA compared to primary tumor and metastasis samples (Figure 3C). Analyzing protein data by western blot (Figure 3D) confirmed the results evaluated on mRNA level. SELENBP1 is stronger expressed in normal skin (NS) compared to melanoma metastases (MM). We furthermore aimed to analyze SELENBP1 by immunohistochemistry experiments on a tissue microarray (TMA) (Figure 3E). Here, ten normal, respectively nevi skin samples, ten primary tumor samples and ten melanoma metastases were spotted on the TMA. Exemplarily, shown are two stainings of each
A

B
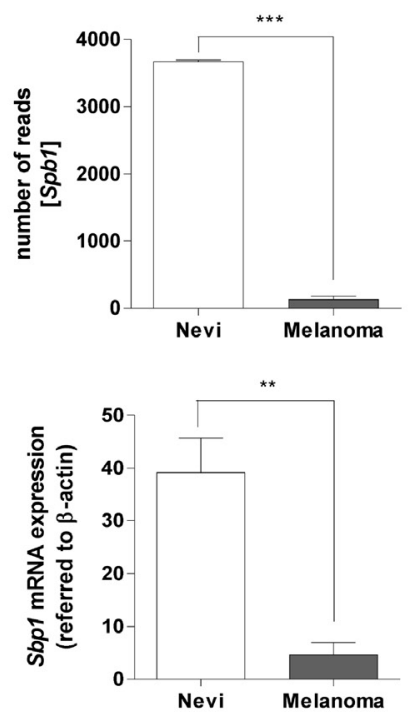

C

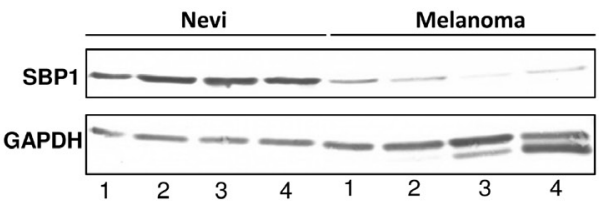

D
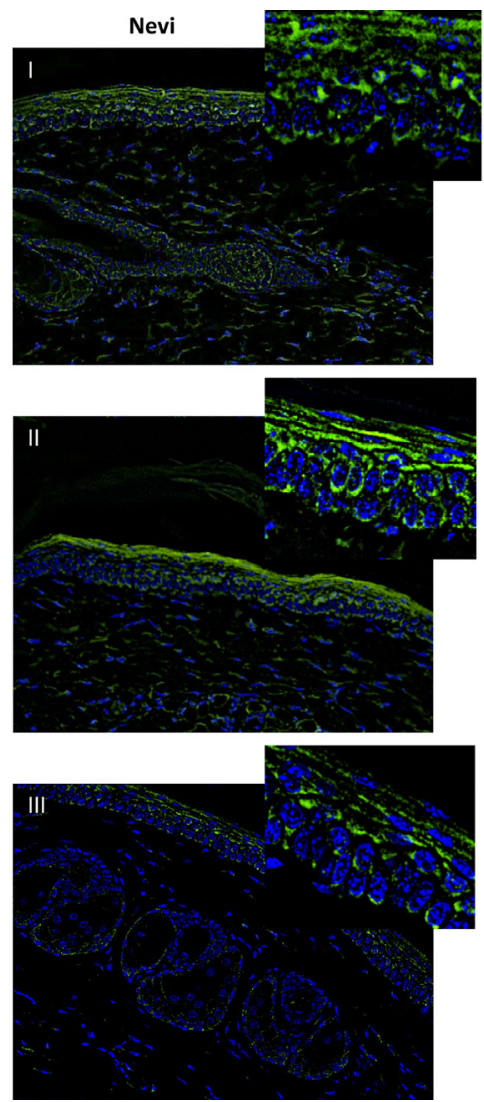
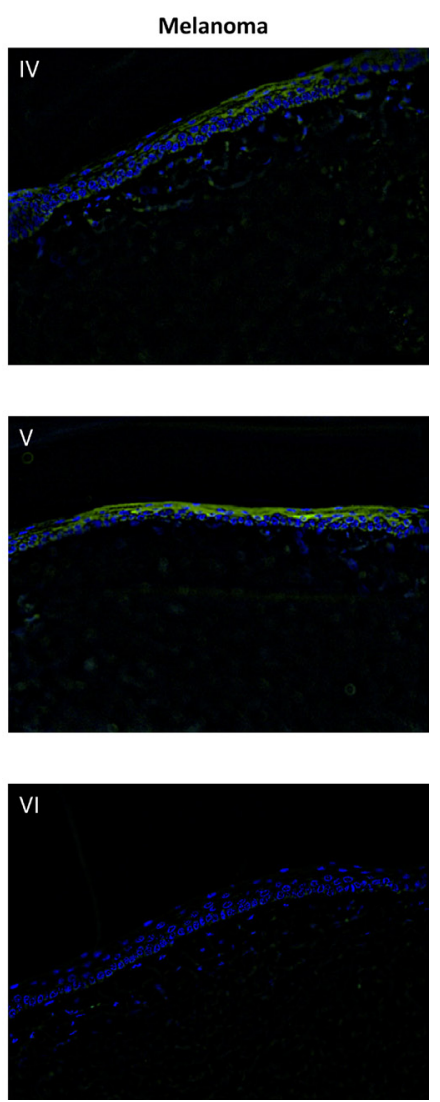

Figure 1: SELENBP1 expression in murine Tg(Grm1) melanoma tissue. (A) SELENBP1 (Sbpl) expression in murine Tg(Grm1) melanoma $(n=2)$ compared to nevi $(n=2)$ tissue via RNA-sequencing analysis. (B) Quantitative real-time PCR analysis to calculate mRNA expression in murine nevi $(n=5)$ and murine melanoma samples $(n=5)\left({ }^{* *} p\right.$-value: 0.0011$)$. (C) Western blot analysis to detect SelenBP1 on protein level in murine melanoma tissue samples $(n=4)$ compared to nevi samples $(n=4)$. GAPDH was used as a loading control. (D) Immunofluorescence analysis of SelenBP1 (green) in murine nevi tissue from the tail (I, II, III) and primary melanomas (IV, $\mathrm{V}, \mathrm{VI})$. DAPI was used to visualize the localization of nuclei. All images showed unspecific green staining of the epidermal keratin layer. 
type. To discriminate between a possible melanin deposit and the brown HRP-detection color we presented also the corresponding H\&E staining of the samples (Figure 3E). The images of specific SELENBP1 (SBP1) staining illustrate again the elevated SELENBP1 expression in normal skin and nevi and the decrease of SELENBP1 in primary melanoma and metastatic melanoma patient tissue. In the corresponding graphical analysis (Figure 3F) we quantified the SELENBP1 staining of the whole TMA. Strong staining was detectable in $66 \%$ of nevi samples by contrast 50 to $80 \%$ of melanoma cases showed SELENBP1 protein staining with low intensity.

\section{SELENBP1 re-expression in human melanoma cells}

To define the role of SELENBP1 in malignant melanoma, the primary melanoma cell line Mel Juso and the metastatic cell line Sk-Mel-28 were transiently transfected with a SELENBP1 expression plasmid (pSBP1). After $24 \mathrm{~h}$, SELENBP1 re-expression was confirmed on both mRNA (Figure 4A) and protein level (Figure 4B). Using the RTCA system neither alterations in cell attachment (Figure 4C), nor changes concerning proliferation (Figure 4D) and migration (Figure 4E), were evident from SELENBP1 re-expression (pSBP1) when compared with pcDNA control-transfected cells. In addition, SELENBP1 re-expression seems to have no impact on self-renewing capacity, as clonogenic forming ability of single cells was not affected (Figure 4F). Moreover, the potential to form vascular-like structures was not influenced by SELENBP1 re-expression, according to results from matrigel-based tube formation assays (Figure 4G). Also in the presence of methylselenic acid (MSA-a selenium metabolite) and $\mathrm{H}_{2} \mathrm{O}_{2}$ (a ROS inducer), clonogenic forming ability of melanoma cells was not altered by transfection with pSBP1 (Figure 4H). In summary, SELENBP1 over-expression had no significant influence on the cancerous behavior of the melanoma cell lines themselves.

The literature suggests the involvement of SELENBP1 in regulating extracellular glutathione (GSH) [29]. We therefore speculated that SELENBP1 overexpression in melanoma cell lines influences the composition of extracellular factors in the medium of the cell lines in culture. Using the supernatant (SN) of pSBP1 transfected cells and transferring it to human dermal microvascular endothelial cells (HMEC) strongly influenced the formation of vascular structures. HMEC cells developed tubes to a lesser and thinner extend using supernatant from SELENBP1 expressing melanoma cells compared to pcDNA control transfected cells (Figure 4I). The expression of specific factors involved in epithelial to mesenchymal transition (EMT) was not affected by the supernatant (SN) of pSBP1 expressing cells, treating keratinocytes (HaCaT), fibroblasts (F V) or HMEC cell lines and analyzing the expression level of Vimentin, $\mathrm{CDH}-1$ and $\mathrm{CDH}-2$ on mRNA level (Supplementary Figure 1C-1E). In summary, SELENBP1 has influence on so far unknown extracellular factors of melanoma cells and influences endothelial cells, like HMECs in a paracrine manner. Although SELENBP1 re-expression has no direct influence on melanoma cells themselves (Figure 4A-4H), we treated SELENBP1 reexpressing melanoma cells with Vemurafenib a kinase inhibitor used in the treatment of patients with unresectable or metastatic melanoma to mimic a "stress" situation of the cells. The combination led to sensitization of Mel Juso melanoma cells for Vemurafenib treatment (Figure 4J).
A

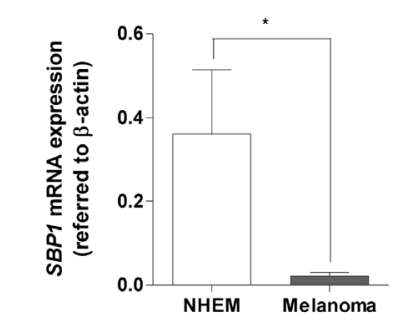

B

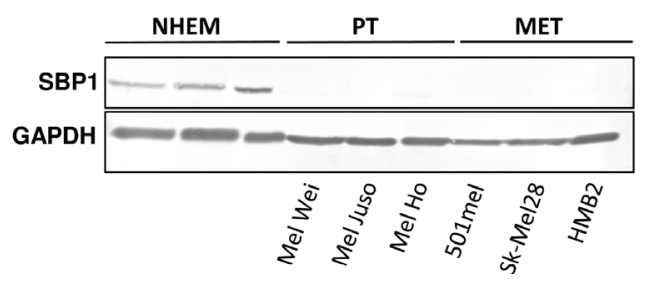

C

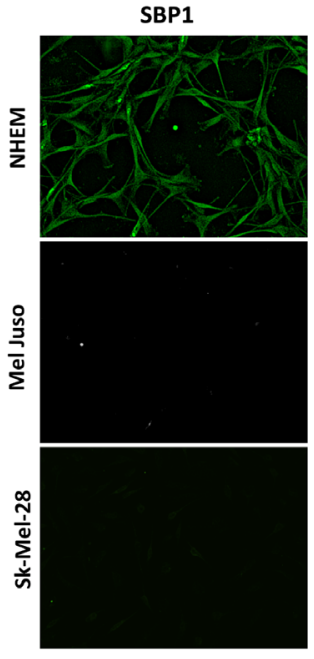

DAPI

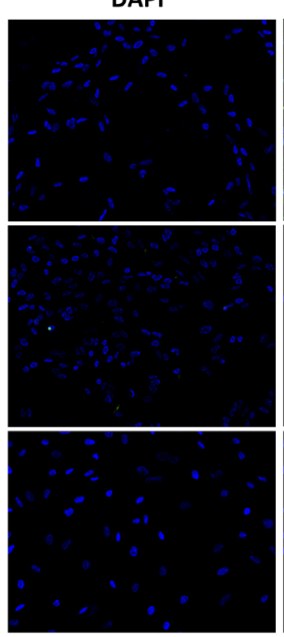

MERGE

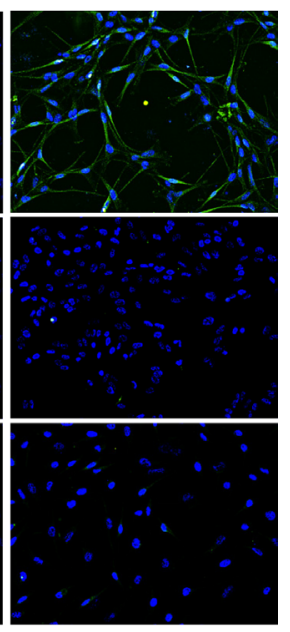

Figure 2: SELENBP1 in human melanoma cell lines. (A) SELENBP1 mRNA expression in melanoma cell lines $(n=10)$ and normal human epidermal melanocytes (NHEM) $(n=8)$. SELENBP1 expression levels were normalized to $\beta$-actin ( ${ }^{*} p$-value: 0.0228$)$. (B) Western blot analysis for detecting SELENBP1 protein in melanoma cell lines and NHEM. GAPDH served as a loading control. (C) Immunofluorescence staining for SELENBP1 protein using representative melanoma cell lines Mel Juso and Sk-Mel-28 and NHEM. DAPI was used for nuclear detection. 


\section{Correlation between SELENBP1 and GPX1}

Geoprofile data revealed significantly elevated levels of GPX1 expression on an mRNA level in malignant melanoma (MET), compared to normal skin (Figure 5A); protein expression data confirmed this finding. Immunohistochemistry showed medium to high expression of GPX1 mainly in the cytoplasm and nucleus (proteinatlas.org) (Figure 5B).

We investigated the expression status of GPX1, another selenium-associated protein shown to be involved in SELENBP1 signaling pathways. Western blot analysis of radial growth phase (RGP), vertical growth phase (VGP), primary tumors which were not classified (PT) and melanoma metastasis (MET) cell lines showed high GPX1 expression in 16 of 18 tested cell lines. 6 of 18 tested melanoma cell lines showed moderate SELENBP1 expression. Both molecules are not expressed in BRAF V600E dependency (Figure 5C). The two melanoma cell lines WM1158 and WM9 showed highest SELENBP1 expression without inverse correlation to GPX1 protein amount.

As it was published in literature that SELENBP1 and GPX1 are influencing each other, directly [24], we transfected melanoma cell lines with siRNA against GPX1 or an over-expression construct for SELENBP1, respectively. Analyzing the expression status on protein and mRNA level, gave no hint either for a direct influence of GPX1 on SELENBP1 expression (Figure 5D) or of SELENBP1 on GPX1 expression (Figure 5E).

Interestingly, we combined re-expression of SELENBP1 and silencing of GPX1 and achieved a significant reduction of proliferation up to $70 \%$ in the cell line Mel Juso (Figure 5F). Sk-Mel-28 only showed a tendency for down-regulated proliferation after a combined manipulation of SELENBP1 and GPX1 expression (Figure 5F). Analyzing the influence of SELENBP1 re-expression and simultaneous GPX1 down-regulation in clonogenic assays led to the result that
A

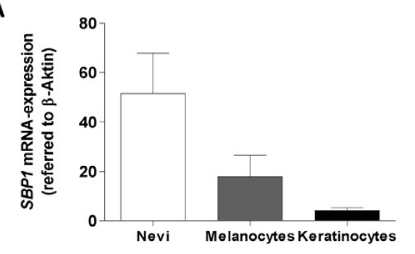

B

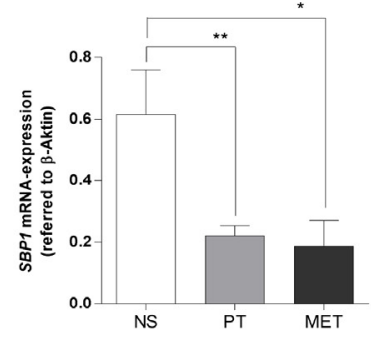

C

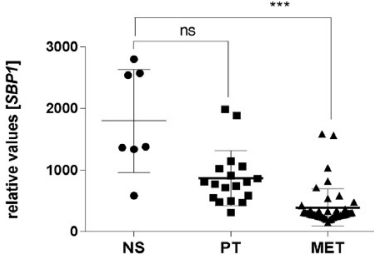

D

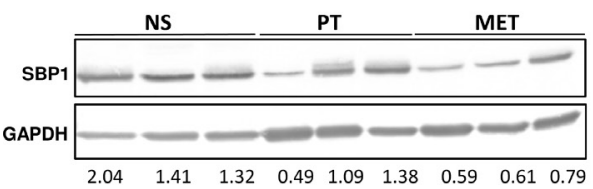

$E$
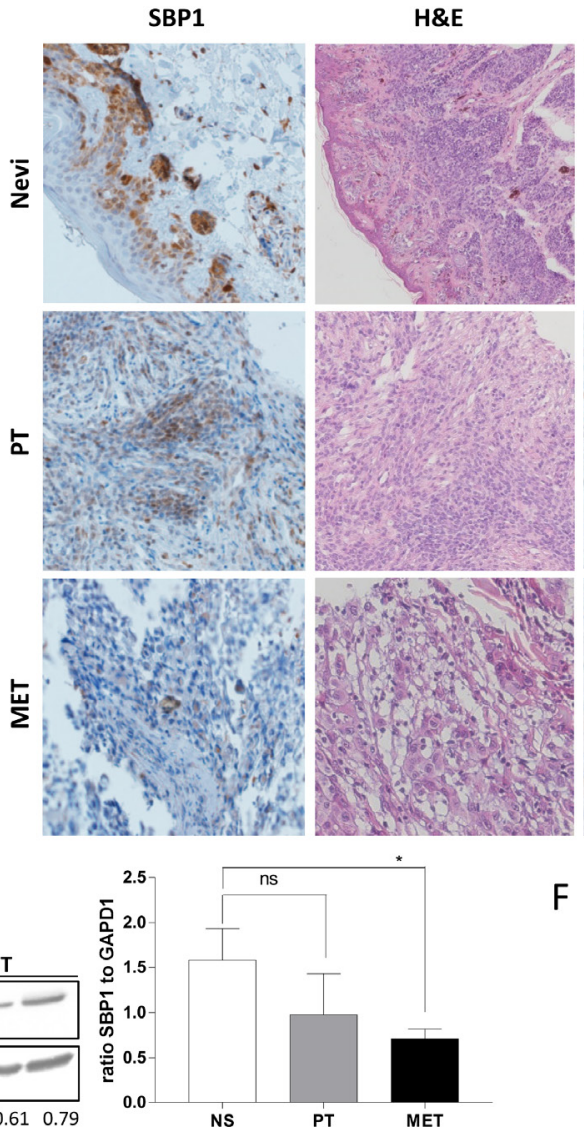
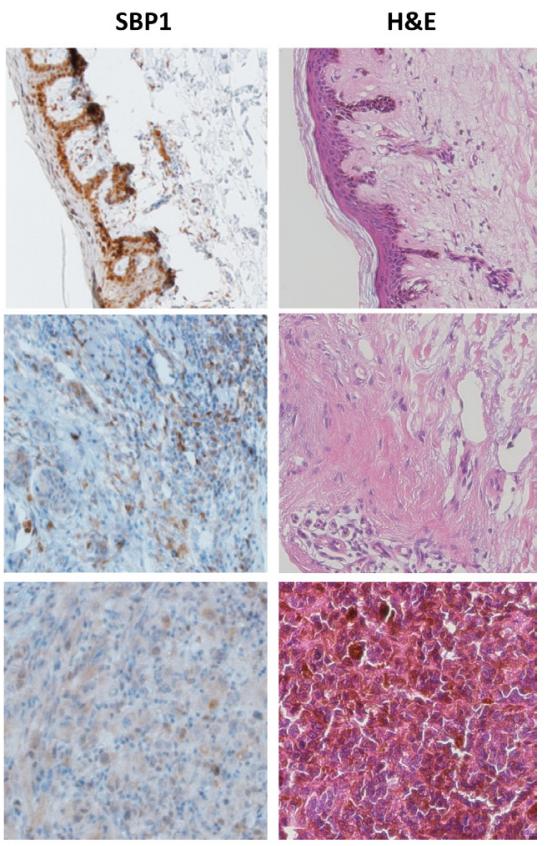

$\mathrm{F}$

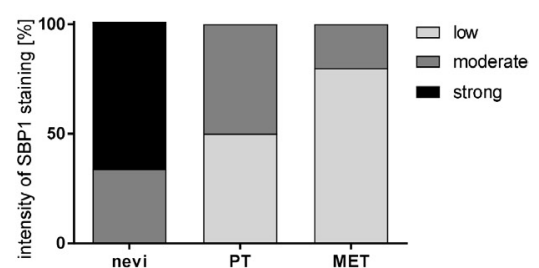

Figure 3: SELENBP1 in vivo in human melanoma (patient). (A) Expression analysis of SELENBP1 in nevi, melanocytes and keratinocytes. (B) Tissue of normal skin $(n=4)$, primary melanoma $(n=4)$ and melanoma metastasis $(n=4)$ were analyzed for SELENBPI expression on mRNA level. (C) Geoprofile data sets (GDS1375) showed SELENPB1 mRNA expression in normal skin (NS) and in primary tumor (PT) and metastasis (MET) of melanoma patient. (D) SELENBP1 protein level was analyzed by Western blot analysis. Values below the blot indicate the ratio between SELENBP1 and GAPDH for each sample and were calculated using ImageJ. (E) Immunohistochemical analysis showed SELENBP1 staining in primary melanoma biopsies $(n=5)$ and melanoma metastases $(n=5)$ compared to nevi tissue $(n=6)$. Representative microscopy images were shown for each tissue, as well as H\&E staining. (F) Statistical analysis of SELENBP1 immunohistochemistry. Evaluation was performed by a classification into three categories: low, moderate and high SELENBP1 protein expression. 
a combination of both molecules reduces the amount of colonies (Figure 5G).

\section{DISCUSSION}

SELENBP1 is suppressed in several human cancers including cancers of the prostate, lung, breast and ovary [11-14]. Indeed, SELENBP1 is described as a tumor suppressor in these tumor types, since decreased levels are associated with enhanced cell proliferation and migration, as well as inhibited apoptosis. Moreover, SELENBP1 reexpression results in diminished cancer cell proliferation and migration, and an induction of apoptosis in colorectal and breast cancer $[13,18,19]$.

Transgenic $\operatorname{Tg}(G r m 1)$ mice serve as a murine model system for spontaneous melanoma development and provide some benefits compared with other melanoma models, avoiding the complication of using and having the feature of metastasis to distant organs including lung and liver [3]. Furthermore, $\operatorname{Tg}(G r m 1)$ mice offer the possibility to compare nevi and melanoma tissue from the same genetic background with respect to changes in the gene expression profile. This mouse model provided the first clues of an important role of SELENBP1 in melanoma and prompted us to further examine this gene in humans. The present study is the first to demonstrate that SELENBP1 is downregulated in human malignant melanoma compared to normal tissue and normal human melanocytes (NHEM). This discovery raises the hypothesis that SELENBP1 functions as tumor suppressor in cutaneous melanoma.

The reason for low or lost, respectively, SELENBP1 expression remains unknown. We excluded $\mathrm{CpG}$ island methylation and loss of function mutations for melanoma and speculate that transcriptional or microRNA dependent suppression is a reason for low SELENBP1 levels. As example, SELENBP1 has been identified as a target of the oxygen-responsive HIF-1 $\alpha$ transcription factor [30].
A

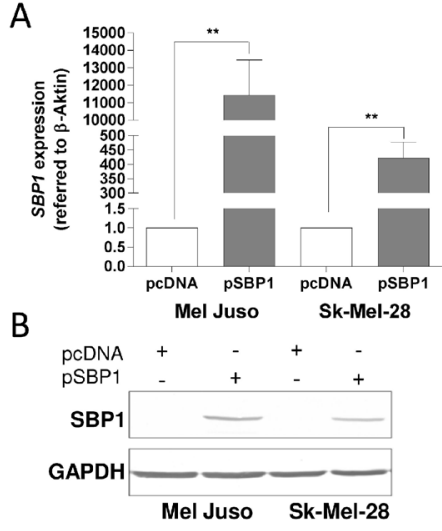

C

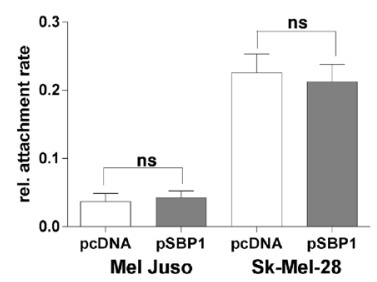

D

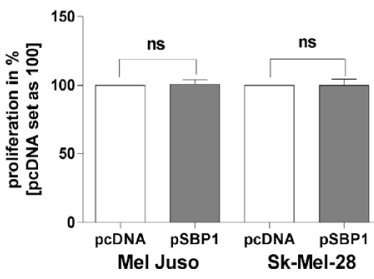

$\mathrm{E}$
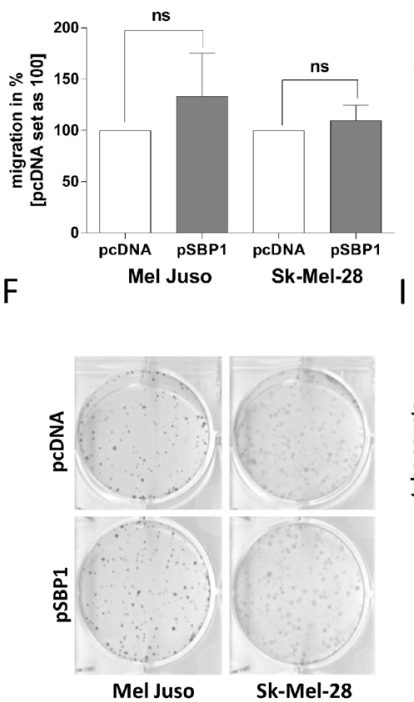

G
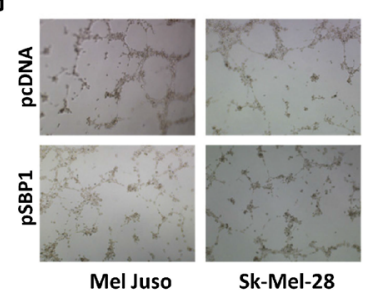

$\mathrm{H}$

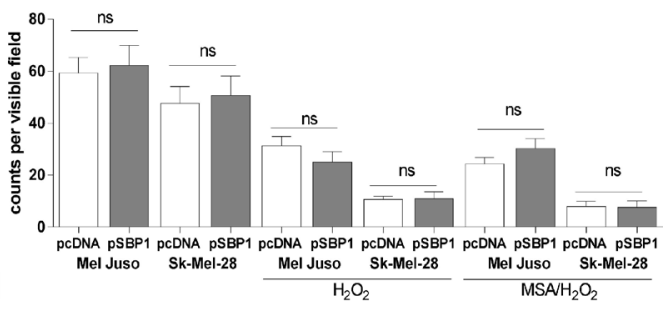

100- HMEC

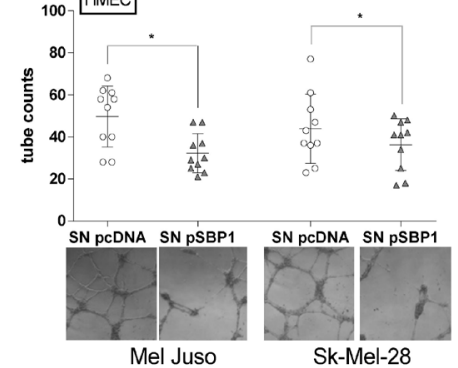

J

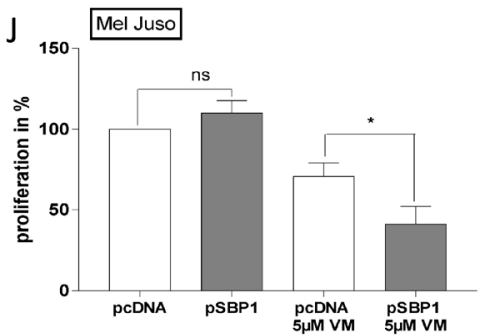

Figure 4: SELENBP1 re-expression in human melanoma cells and cellular mechanisms. (A) Quantitative real-time PCR analysis and (B) Western blot analysis confirmed SELENBP1 (pSBP1) re-expression in Mel Juso and Sk-Mel-28 melanoma cell lines, compared to pcDNA control-transfected cells $24 \mathrm{~h}$ after transfection $\left({ }^{* *} p<0.01\right)$. (C-E) RTCA experiments to analyze alterations in cell attachment, proliferation and migration (ns: not significant). (F) Clonogenic assays for analyzing the impact of SELENBP1 re-expression on self-renewing capacity. (G) Matrigel-based tube formation assays displayed the development of vascular channels after transfection with pSBP1 vector. (H) MSA and $\mathrm{H}_{2} \mathrm{O}_{2}$ treatment together with pcDNA or SELENBP1 (pSBP1) over-expression, respectively, was analyzed by RTCA (ns: not significant). (I) Supernatant (SN) of melanoma cell lines re-expressing SELENBP1 (pSBP1) and control transfected cells (pcDBNA) was used for cell culture of human dermal microvascular endothelial cells (HMECs). Matrigel-based tube formation assays displayed the vessel formation. (J) RTCA proliferation assay for the melanoma cell line Mel Juso treated with Vemurafenib (5 $\mu \mathrm{M})$ and transfected with the SELENBP1 expression construct, respectively. 
To characterize the consequences of SELENBP1 suppression in malignant melanoma several functional assays were performed using a human SELENBP1 expression plasmid (pSBP1). However, these in vitro analyses gave no evidence that SELENBP1 has a functional impact on melanoma cells themselves in terms of attachment, proliferation, migration, self-renewing capacity and tube formation. As reported by others, an inhibitory effect of SELENBP1 re-expression on proliferation in cell culture may require the MSA (a selenium metabolite) supplementation to ensure sufficient selenium in the assays [18]. As example, breast cancer cells without MSA treatment did not display altered proliferation potential upon transfection with a SELENBP1 plasmid [11]. Therefore, we incubated melanoma cells also with MSA and again detected no influence by combination of SELENPB1 re-expression and simultaneous MSA treatment. Additional studies report that altered proliferation in SELENBP1 dependency occurs only after treatment with hydrogen peroxide [20, 23]; $\mathrm{H}_{2} \mathrm{O}_{2}$ is a reactive oxygen species (ROS) leading to enhanced oxidative stress in cells. Enhanced ROS level can induce DNA damage of tumor cells and lead to cell death, requiring cancer cells to adopt protective mechanisms. These hints from the literature prompted us to test MSA and $\mathrm{H}_{2} \mathrm{O}_{2}$ treatment in human malignant melanoma cells. Neither MSA nor $\mathrm{H}_{2} \mathrm{O}_{2}$ treatment altered the effects of SELENBP1 transfection in malignant melanoma cells in our experiments. To proof, whether SELENBP1 exerts influences on Vemurafenib treatment of melanoma cells we incubated cells with Vemurafenib and re-expressed SELENBP1, which sensitized melanoma cells for the therapy.

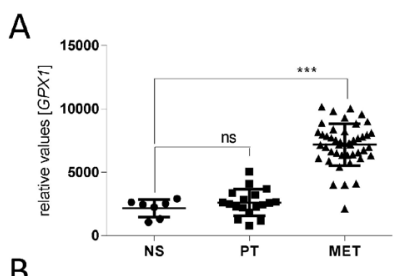

B

\begin{tabular}{|l|l|l|l|l|l|}
\hline \multicolumn{2}{|l|}{$\begin{array}{l}\text { Antibody staining } \\
{[10]}\end{array}$} & \multicolumn{2}{l|}{$\begin{array}{l}\text { Intensity } \\
{[10]}\end{array}$} & \multicolumn{2}{l|}{ Localization } \\
\hline high & 1 & strong & 1 & $\mathrm{c} / \mathrm{m} / \mathrm{n}$ & 0 \\
\hline medium & 8 & moderate & 8 & $\mathrm{c} / \mathrm{m}$ & 10 \\
\hline low & 1 & weak & 1 & $\mathrm{n}$ & 0 \\
\hline negativ & 0 & negativ & 0 & none & 0 \\
\hline
\end{tabular}

C
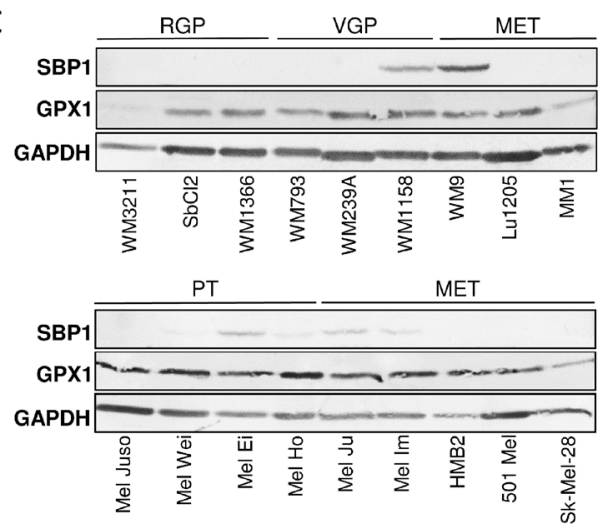
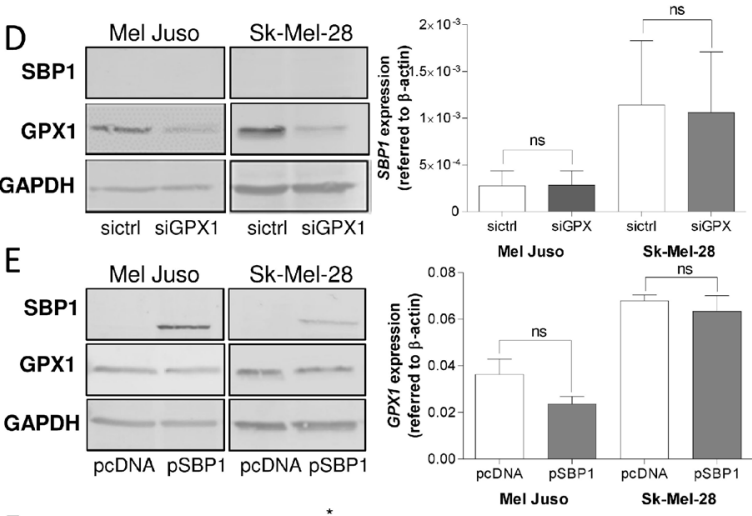

$\mathrm{F}$

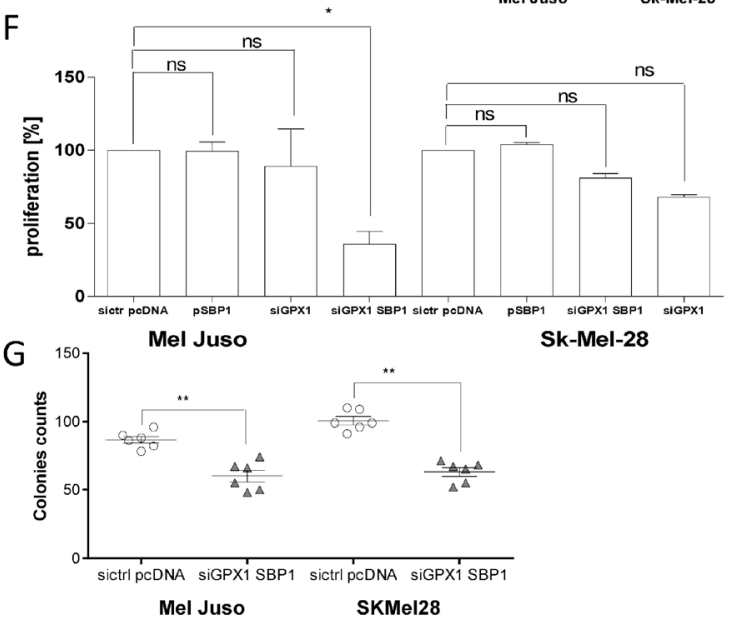

Figure 5: Connection between SELENBP1 and GPX1.(A) Geoprofile data sets (GDS1375) show GPX1 mRNA expression in normal skin (NS), primary tumor (PT) and metastasis (MET) of melanoma patients $\left({ }^{* * *} p<0.001\right.$; ns, not significant). (B) Immunohistochemical analysis showing GPX1 staining in human melanoma samples ( $n=10$, data bank of proteinatlas.org). Representative microscopy images are shown. Evaluation was performed by classification into three categories: low, medium and high GPX1 protein expression. (C) Western blot analysis of SELENBP1 and GPX1 protein in 18 different melanoma cell lines of different tumor stages (RGP: radial growth phase; VGP: vertical growth phase; PT: primary tumor; MET: metastasis). GAPDH was used as a loading control. (D) Treatment of cells with siRNA against GPX1 and confirmation of successful knock-down by using anti-GPX1 antibody. Analysis of the influence of silenced GPX1 on SELENBP1 expression using an anti-SELENBP1 antibody and analyzing the SELENBP1 expression on mRNA level. (E) Treatment of cells with a re-expression vector (pSBP1) for SELENBP1 and confirmation of successful re-expression by using anti-SELENBP1 antibody. Analysis of the influence of re-expressed SELENBP1 on GPX1 expression using an anti-GPX1 antibody and analyzing the GPX1 expression on mRNA level. (F) RTCA proliferation assay after SELENBP1 re-expression of GPX1 silencing alone and in combination. (G) Clonogenic assays for analyzing the impact of SELENBP1 re-expression and GPX1 knock-down on self-renewing capacity. 
Loss of SELENBP1 resulted in the high-affinity uptake of selenite through elevating the levels of extracellular GSH. Reduction of SBP1 accelerated uptake of extracellular selenite and reduction of SBP1 enhanced selenite-mediated cytotoxic effects through elevating extracellular GSH levels [29] with possible consequences also of the vessel formation of endothelial cells in the environment of cancer cells. We therefore speculated that SELENBP1 overexpression in melanoma cell lines influences the composition of extracellular factors thus influencing the tumor microenvironment. Interestingly, supernatant taken from melanoma cell lines transfected with a SELENBP1 re-expression plasmid led to suppression of vessel formation of HMEC cells.

Glutathione peroxidase (GPX1), an intracellular antioxidant enzyme, is a protector against ROS effects [31]. Interestingly, elevated GPX1 activity causes an increase in cell proliferation [21]. In previous studies, increasing the levels of SELENBP1 reduced GPX1 enzyme activity $[20,23,24]$ and reducing SELENBP1 levels increased GPX1 enzyme activity. In summary, it was speculated that SELENBP1 inhibits GPX1 activity and thus may function as a tumor suppressor only by regulating GPX1. Our results did not confirm these results for melanoma. Manipulating GPX1 expression did not influence SELENBP1 mRNA and protein amount and vice versa. The genes are not regulating each other and loss of SELENBP1 and over-expression of GPX1 eventually are independent events during melanoma progression. We speculate that both molecules still compensate the function from each other.

However, simultaneous SELENBP1 overexpression plus GPX1 knockdown reduces melanoma cell proliferation significantly. These results support that SELENBP1 could be a tumor suppressor lost early in development in melanoma and it was functionally compensated by GPX1 in later melanoma progression.

In summary, our results show that data obtained from the $\operatorname{Tg}(G r m 1)$ mouse model are relevant to the human system and may provide insights into molecular modifications leading to melanoma development and progression. Therefore, this innovative model system is a useful tool for unraveling new genes involved in melanomagenesis. Furthermore, SELENBP1 re-expression leads to changes in proliferation of melanoma cells when incubating it together with Vemurafenib. Additionally, we revealed that SELENBP1 changes microenvironmental factors and has paracrine influence on surrounding cell types of melanoma cells, as shown for human dermal microvascular endothelial cells (HMEC).

\section{MATERIALS AND METHODS}

\section{Transgenic mice}

The transgenic $\operatorname{Tg}(\mathrm{Grm} 1)$ mice were established at the Department of Chemical Biology, Rutgers University,
Piscataway, USA [2] and kindly provided by Prof. Suzie Chen and Prof. Jürgen Becker. Mice were kept under standard conditions at $21^{\circ} \mathrm{C}\left( \pm 1^{\circ} \mathrm{C}\right)$ with $55 \%( \pm 10 \%)$ relative humidity and $12 \mathrm{~h}$ light/dark intervals. Animals were fed with standard chow (Ssniff, Soest, Germany) and with drinking water ad libitum [3]. Animal care and experimental procedures were carried out in accordance with the guidelines of the German law governing animal care. Experiments were approved by the Ethics Committee for Animal Research of the Bavarian government. For all analyses, we used homozygous transgenic animals bred in our laboratory.

\section{Cell culture and tissue samples}

Human melanoma cell lines Mel Ei, Mel Wei, Mel Juso, Mel Ho (derived from primary cutaneous melanoma), Mel Ju, Mel Im, 501 Mel, Sk-Mel-28 and $\mathrm{Hmb} 2$ (derived from metastases of malignant melanoma) were cultured in Dulbecco's modified Eagle's medium (DMEM) supplemented with penicillin (400 U/ml), streptomycin $(50 \mu \mathrm{g} / \mathrm{ml})$ and $10 \%$ fetal calf serum (all from Sigma-Aldrich, München, Germany). Normal human epidermal melanocytes (NHEM) were cultivated in melanocyte growth medium M2 (PromoCell, Heidelberg, Germany). HMEC cells were cultivated in Medium 200 (Gibco, Thermo Fisher Scientific, Rockford, USA) supplemented with 50× LVES (Gibco) and penicillin $(400 \mathrm{U} / \mathrm{ml})$, streptomycin $(50 \mu \mathrm{g} / \mathrm{ml})$. $\mathrm{HaCaT}$ and FV cells were cultivated in DMEM supplemented with penicillin $(400 \mathrm{U} / \mathrm{ml})$, streptomycin $(50 \mu \mathrm{g} / \mathrm{ml})$ and $10 \%$ fetal calf serum. All cell lines were incubated at $37^{\circ} \mathrm{C}$ in a humidified atmosphere containing $8 \% \mathrm{CO}_{2}$.

$\mathrm{H}_{2} \mathrm{O}_{2}$ treatment was performed at a concentration of $25 \mu \mathrm{M}$. Methylseleninic acid (MSA) was dissolved and added to the cell cultures as described by Liu et al. [32]. Vemurafenib was purchased by Absource Diagnostics (Munich, Germany). Human tissue samples of snapfrozen normal skin (TB 20, TB30, TB33, TB34), nevi, primary melanoma tumors (TB97, TB148, TB199) and melanoma metastases (Met124, Met202, Met203) with clear-cut pathological classification were obtained from our tissue collection (Institute of Pathology, University of Regensburg, Germany). Sampling and handling of patient material were carried out in accordance with the ethical principles of the Declaration of Helsinki.

\section{Transfection experiments}

Cells were plated $2 \times 10^{5}$ cells/well into 6-well plates and transfected with $0.5 \mu \mathrm{g}$ plasmid DNA using the lipofectamine plus method (Invitrogen, Darmstadt, Germany), according to the manufacturer's instructions. The human SELENBP1 expression construct (pSBP1) was provided by the research group of Prof. W. Yang [19]. 
SiRNA against GPX1 was purchased from Qiagen (Hilden, Germany) and used in a concentration of $20 \mu \mathrm{M}$ (stock).

\section{Treatment of cell with 5-Aza-2'-deoxycytidine}

The melanoma cell lines were seeded at a low density of 750,000 cells in a T75 flask, $24 \mathrm{~h}$ before treatment. The next day cells were treated with $5 \mu \mathrm{M}$ 5-Aza-2'-deoxycytidine (Sigma-Aldrich; dissolved in $50 \%$ acetic acid, diluted in DMEM/10\% FCS) for $72 \mathrm{~h}$. Control cells were incubated with the same volume of acetic acid diluted to a $50 \%$ solution with PBS also for the time period of $72 \mathrm{~h}$. Following this incubation, $300 \mathrm{nM}$ Trichostatin A (TSA, Sigma Aldrich) was added to fresh media for $4 \mathrm{~h}$. The control cells were incubated with the same volume of PBS diluted in fresh media.

\section{Hypoxia}

Membrane permeable Desferrioxamine (DFX) and 2, 2'-dipyridyl (DP) (purchased from SigmaAldrich) as iron chelators and inhibitors of Prolylhydroxylases were used in a concentration of $250 \mu \mathrm{M}$ for DFX and $50 \mu \mathrm{M}$ for DP diluted in DMEM.

\section{Measurement of migration, attachment, and proliferation}

The xCELLigence System (Roche, Mannheim, Germany) is based on measurement of electrical impedance and permits real-time analysis of migration, attachment, and proliferation. CIM plates (migration) and E-plates (attachment and proliferation) were used and basic protocols recommended by the manufacturer were followed. The bottom chambers contained culture supernatant from human fibroblasts as chemo-attractant. Upper chambers contained serum-free DMEM. After recording background impedance, cells suspended in serum-free DMEM were added to the upper chambers ( $4 \times 10^{3} /$ well for migration; $2 \times 10^{2} /$ well for proliferation/ attachment). Thereafter, impedance can be measured continuously over $72 \mathrm{~h}$ or longer. Impedance is represented by the relative and dimensionless parameter named cell index $(\mathrm{CI})$. CI values $=\mathrm{Zi}-\mathrm{Z0} / 15[\mathrm{Ohm}]$; where $\mathrm{Z} 0=$ impedance at the start of the experiment, and $\mathrm{Zi}=$ impedance at individual time points during the experiment. The normalized cell index (NCI) was calculated as the cell index $\mathrm{CI}_{\mathrm{ti}}$ at a given time point (ti) divided by the cell index $\mathrm{CI}_{\text {nml_time }}$ at the normalization time point (nml_time). The slope is used to describe the steepness of a curve within a given time window (in our case $1.5 \mathrm{~h}$ (attachment) $4 \mathrm{~h}$ (migration, and $100 \mathrm{~h}$ (proliferation)).

\section{Clonogenic assay (stem cell behavior)}

We used the clonogenic assay as the method of choice to test the survival rate based on the ability of a single cell to grow into a colony. The assay essentially tests every cell in the population for its ability to undergo "unlimited" division. The in vitro assay performed as described by Franken et al. [33]. 50 or 100 cells, respectively, were sowed in a 6-well chamber, cultivated for approximately 10 days to a colony size of $\sim 50$ cells. The colonies were counted by microscopy.

\section{Tube formation (vasculogenic mimicry)}

Growth factor reduced Matrigel (BD Biosciences, Heidelberg, Germany) was added to eight-chamber polystyrene vessel tissue culture-treated glass slides (BD Bioscience) and allowed to gelatinize for $20 \mathrm{~min}$ at $37^{\circ} \mathrm{C}$. To assay vasculogenic mimicry, $7 \times 10^{4}$ melanoma cells or HMEC cells, respectively were seeded onto Matrigelcoated culture slides. Tube formation was assessed by phase contrast microscopy after $16 \mathrm{~h}$ and recorded with a digital camera.

\section{RNA isolation, reverse transcription and quantitative RT-PCR}

Total RNA was isolated using the E.Z.N.A. MicroElute Total RNA Kit (Omega Bio-Tek, VWR Darmstadt, Germany) according to the manufacturer's instructions. For RNA isolation of tissue samples ceramic beads and the Precellys homogenisator (Peqlab Biotechnologies GmbH, Erlangen, Germany) were used for mechanical fragmentation. RNA concentration was measured with a NanoDrop spectrophotometer (Peqlab Biotechnology $\mathrm{GmbH}$ ) and cDNA was generated by reverse transcription using the Super Script II Reverse Transcriptase Kit (Life Technologies, Carlsbad, USA), with each reaction containing $500 \mathrm{ng}$ of total RNA. Analysis of mRNA expression was performed using quantitative Real-Time PCR on the LightCycler 480 system (Roche, Mannheim, Germany). A volume of $1 \mu \mathrm{l}$ cDNA template, $0.5 \mu \mathrm{l}$ of forward and reverse primers (each $20 \mu \mathrm{M}$ ) and $10 \mu \mathrm{l}$ of SYBR Green I (Roche, Mannheim, Germany) were combined to a total volume of $20 \mu \mathrm{l}$. The following primers were used: $h \beta$-Actin for $5^{\prime}$ TGACGGGGTCACCCAC AC-3'; h $\beta$-Actin rev 5'-TAAAACGCAGCTCAGTAACAG TCCG-3'; m $\beta$-Actin for 5'-TGGAATCCTGTGGCAT CCATGAAAC-3'; m $\beta$-Actin rev 5'-TAAAACGCAGCT CAGTAACAGTCCG-3'; hSELENBP1 for 5'-ATCTGG CCACTGTGGATGTT-3'; hSELENBP1 rev 5'-CACCAC ATAGATGCGAGAGGA-3'; mSELENBP1 for 5'-GCAC TGAAGCCCCGGATTAT-3'; mSELENBP1 rev 5'-ACATC CACCACGTAGATGCG-3'; hGPX1 for 5'-CGCC AAGAACGAAGAGATTC-3'; hGPX1 rev 5'-AAAGT TCCAGGCAACATCGT-3'; hCDH-1 for 5'-ACCAGGAC TTTGACTTGAGC-3'; hCDH-1 rev 5'-GACTAGCAGC TTCGGAACC-3'; hCDH-2 for5'-TGGATGAAGATGG CATGG-3'; hCDH-2 rev5'-AGGTGGCCACTGTGCTTAC 
-3'; hVimentin for 5'-TGGCCGACGCCATCAACACC3'; hVimentin rev 5'-CACCTCGACGCGGGCTTTGT-3'. Each sample was analyzed in duplicate. The target cDNA was normalized to $\beta$-actin levels.

\section{RNA-sequencing}

For gene expression analysis RNA samples of two nevi and two melanoma samples from $\operatorname{Tg}(\mathrm{Grm} /)$ mice were analyzed by RNA-sequencing. For this purpose, four poly-A RNA sequence libraries were generated. Single-end reads of $100 \mathrm{bp}$ were sequenced at the Center of Excellence for Fluorescent Bioanalytics (KFB) (Regensburg, Germany; http://www.kfb-regensburg.de) with HiScanSQ (TruSeq SBS kit v3; Illumina, San Diego, CA, USA) technology from Illumina. Sample reads were aligned to the mouse reference genome $\mathrm{mm} 9$ (NCBI37) from UCSC (University of California, Santa Cruz, CA, USA) using Rsubread [34] with the default parameters and the in-built annotation data for $\mathrm{mm} 9$. The sequence data are available at NCBI, BioProject PRJNA237546. Gene counts based on Entrez genes (http://www.ncbi.nlm. nih.gov/gene) were generated with feature Counts [34] using default parameters for single end data. Differential gene expression was performed using edgeR [35] Genes with a false discovery rate below 0.01 were considered significantly differentially expressed.

\section{Protein isolation and western blot analysis}

Cells and tissues were lysed in $200 \mu$ RIPA buffer (Roche, Mannheim, Germany) for $15 \mathrm{~min}$ at $4^{\circ} \mathrm{C}$ and cell debris was separated via centrifugation at 13,000 rpm and $4^{\circ} \mathrm{C}$ for $10 \mathrm{~min}$. Protein concentration was determined using the Pierce BCA Protein Assay Kit (Thermo Fisher Scientific, Rockford, USA). For each sample, $40 \mu \mathrm{g}$ of total lysate were separated on 10\% SDS-PAGE gels and subsequently transferred onto a PVDF membrane. After blocking for $1 \mathrm{~h}$ with $5 \% \mathrm{BSA} / \mathrm{PBS}$ the membrane was incubated overnight $\left(4^{\circ} \mathrm{C}\right)$ with one of the following antibodies: anti-SELENBP1 (Abcam, Cambridge; 1:1000), anti- $\beta$-actin (Sigma-Aldrich, Missouri, USA; 1:5000), anti-GXP1 (Thermo Fisher Scientific; 1:1000) or anti-GAPDH (Cell Signaling Technology, Frankfurt a.M.; 1:1000). After washing three times with TBS-T, the membrane was probed with an alkaline phosphatecoupled secondary antibody (anti-rabbit AP or anti-mouse AP, Cell Signaling Technology, Frankfurt a.M., Germany; 1:4000 and 1:3000, respectively) for $1 \mathrm{~h}$. Finally, the membrane was washed three times with TBS-T and the immunoreaction was visualized using NBT/BCIP (Life technologies, Carlsbad, USA).

\section{Immunohistochemical analysis}

Standard $5 \mu \mathrm{m}$ sections of formalin-fixed and paraffin-embedded tissue blocks were used for immunohistochemistry of murine and human tissue samples. Immunohistochemical staining was performed using anti-SELENBP1 antibody (Abcam, Cambridge, UK; 1:100) and the Envision ${ }^{\mathrm{TM}}$ system (Dako, Hamburg, Germany) for human and the Permanent HRP Green Kit (Zytomed, Berlin, Germany) for murine tissue samples. Immunofluorescence staining of cells was performed from each cell line seeded $\left(5 \times 10^{4}\right.$ cells $)$ onto eightwell chamber slides (Corning Incorporated, Corning, USA) and incubated overnight at $37^{\circ} \mathrm{C}$. After $15 \mathrm{~min}$ fixation with 4\% PFA, one washing step with PBS, 5 min, incubation with $0.1 \%$ Triton-X-100 followed. Subsequently, cells were washed three times with PBS and blocked for $1 \mathrm{~h}$ with $1 \% \mathrm{BSA} / \mathrm{PBS}$. Incubation with the anti-SELENBP1 antibody (Abcam, Cambridge, UK; $1: 120$ ) continued over night at $4^{\circ} \mathrm{C}$, and after three washing steps the secondary Alexa Fluor 488 anti-rabbit antibody (Life technologies, Carlsbad, USA; 1:500) was added for $1 \mathrm{~h}$. Finally, cells were washed again with PBS and VECTASHIELD ${ }^{\mathrm{TM}}$ Slide Mounting Medium with DAPI (Vector Laboratories Inc., Burlingame, USA) was added for mounting.

\section{Statistical analysis}

Results are shown as the mean \pm standard error of the mean or percent, and statistical significance was determined using the Student's unpaired $t$-test (Figures 1, 2, 3). Comparison between more than two groups was made using a one-way ANOVA (Kruskal-Wallis test) analysis of variance (Figure 4 and 5) calculated with GraphPad Prism 7 (GraphPad Software, Inc., San Diego, USA). A p-value $<0.05$ was considered as statistically significant (ns: not significant, ${ }^{*} p<0.05,{ }^{* *} p<0.01$ ).

\section{Abbreviations}

Selenium-binding protein 1: SELENBP1; Glutathione peroxidase 1, GPX1: normal human epidermal melanocytes, NHEM; NS: normal skin; PT: primary tumor; MET: melanoma metastasis; MSA: methylseleninic acid.

\section{Authors contributions}

MS, MMdJ and PR were responsible for the practical work, JCE for the bioinformatics, EKG, AKB and SK for the project design and text.

\section{ACKNOWLEDGMENTS}

We thank Prof. Suzie Chen (Rutgers University, Chemical Biology, Piscataway, USA) and Prof. Jürgen Becker (University Duisburg-Essen, Germany) for providing $\operatorname{Tg}(\mathrm{Grml})$ animals, Prof. Wancai Yang for providing the hSELENBP1 plasmid (pSBP1) and Rudolf Jung for excellent technical assistance. 


\section{CONFLICTS OF INTEREST}

The authors declare no conflicts of interest.

\section{FUNDING}

This study was supported by the German Research Foundation (DFG, Research group FOR2127), German Cancer Aid, and BioSysNet.

\section{REFERENCES}

1. Martín García E, Arias-Santiago S, Serrano-Ortega S, Buendía-Eisman A. [Changes in the Incidence of Skin and Lip Cancer Between 1978 and 2007.] [Article in Spanish]. Actas Dermosifiliogr. 2017; 108:335-45.

2. Pollock PM, Cohen-Solal K, Sood R, Namkoong J, Martino JJ, Koganti A, Zhu H, Robbins C, Makalowska I, Shin SS, Marin Y, Roberts KG, Yudt LM, et al. Melanoma mouse model implicates metabotropic glutamate signaling in melanocytic neoplasia. Nat Genet. 2003; 34:108-112.

3. Schiffner S, Braunger BM, de Jel MM, Coupland SE, Tamm ER, Bosserhoff AK. $\operatorname{Tg}(\mathrm{Grm} 1)$ transgenic mice: a murine model that mimics spontaneous uveal melanoma in humans? Exp Eye Res. 2014; 127:59-68.

4. Namkoong J, Shin SS, Lee HJ, Marin YE, Wall BA, Goydos JS, Chen S. Metabotropic glutamate receptor 1 and glutamate signaling in human melanoma. Cancer Res. 2007; 67:2298-2305.

5. Lee HJ, Wall BA, Wangari-Talbot J, Chen S. Regulation of mGluR1 expression in human melanocytes and melanoma cells. Biochim Biophys Acta. 2012; 1819:1123-1131.

6. de Jel MM, Engelmann JC, Kunz M, Schiffner S, Kuphal S, Bosserhoff AK. Transcriptome sequencing of melanocytic nevi and melanomas from Grm1 transgenic mice to determine melanoma driver mutations. Pigment Cell Melanoma Res. 2014; 27:678-680.

7. Irons R, Carlson BA, Hatfield DL, Davis CD. Both selenoproteins and low molecular weight selenocompounds reduce colon cancer risk in mice with genetically impaired selenoprotein expression. J Nutr. 2006; 136:1311-1317.

8. Peters U, Littman AJ, Kristal AR, Patterson RE, Potter JD, White E. Vitamin E and selenium supplementation and risk of prostate cancer in the Vitamins and lifestyle (VITAL) study cohort. Cancer Causes Control. 2008; 19:75-87.

9. Peters U, Takata Y. Selenium and the prevention of prostate and colorectal cancer. Mol Nutr Food Res. 2008; 52:1261-1272.

10. Klein EA. Selenium: epidemiology and basic science. J Urol. 2004; 171:S50-53.

11. Ansong E, Ying Q, Ekoue DN, Deaton R, Hall AR, Kajdacsy-Balla A, Yang W, Gann PH, Diamond AM.
Evidence that selenium binding protein 1 is a tumor suppressor in prostate cancer. PLoS One. 2015; 10:e0127295.

12. Chen G, Wang H, Miller CT, Thomas DG, Gharib TG, Misek DE, Giordano TJ, Orringer MB, Hanash SM, Beer DG. Reduced selenium-binding protein 1 expression is associated with poor outcome in lung adenocarcinomas. $\mathrm{J}$ Pathol. 2004; 202:321-329.

13. Zhang C, Xu W, Pan W, Wang N, Li G, Fan X, Xu X, Shen S, Das UN. Selenium-binding protein 1 may decrease gastric cellular proliferation and migration. Int J Oncol. 2013; 42:1620-1629.

14. Huang KC, Park DC, Ng SK, Lee JY, Ni X, Ng WC, Bandera CA, Welch WR, Berkowitz RS, Mok SC, Ng SW. Selenium binding protein 1 in ovarian cancer. Int J Cancer. 2006; 118:2433-2440.

15. Li T, Yang W, Li M, Byun DS, Tong C, Nasser S, Zhuang M, Arango D, Mariadason JM, Augenlicht LH. Expression of selenium-binding protein 1 characterizes intestinal cell maturation and predicts survival for patients with colorectal cancer. Mol Nutr Food Res. 2008; 52:1289-1299.

16. Silvers AL, Lin L, Bass AJ, Chen G, Wang Z, Thomas DG, Lin J, Giordano TJ, Orringer MB, Beer DG, Chang AC. Decreased selenium-binding protein 1 in esophageal adenocarcinoma results from posttranscriptional and epigenetic regulation and affects chemosensitivity. Clin Cancer Res. 2010; 16:2009-2021.

17. Zhang J, Dong WG, Lin J. Reduced selenium-binding protein 1 is associated with poor survival rate in gastric carcinoma. Med Oncol. 2011; 28:481-487.

18. Zhang S, Li F, Younes M, Liu H, Chen C, Yao Q. Reduced selenium-binding protein 1 in breast cancer correlates with poor survival and resistance to the anti-proliferative effects of selenium. PLoS One. 2013; 8:e63702.

19. Pohl NM, Tong C, Fang W, Bi X, Li T, Yang W. Transcriptional regulation and biological functions of selenium-binding protein 1 in colorectal cancer in vitro and in nude mouse xenografts. PLoS One. 2009; 4:e7774.

20. Huang C, Ding G, Gu C, Zhou J, Kuang M, Ji Y, He Y, Kondo T, Fan J. Decreased selenium-binding protein 1 enhances glutathione peroxidase 1 activity and downregulates HIF1alpha to promote hepatocellular carcinoma invasiveness. Clin Cancer Res. 2012; 18:3042-3053.

21. Faucher K, Rabinovitch-Chable H, Cook-Moreau J, Barriere G, Sturtz F, Rigaud M. Overexpression of human GPX1 modifies Bax to Bcl-2 apoptotic ratio in human endothelial cells. Mol Cell Biochem. 2005; 277:81-87.

22. de Haan JB, Bladier C, Griffiths P, Kelner M, O'Shea RD, Cheung NS, Bronson RT, Silvestro MJ, Wild S, Zheng SS, Beart PM, Hertzog PJ, Kola I. Mice with a homozygous null mutation for the most abundant glutathione peroxidase, Gpx1, show increased susceptibility to the oxidative stressinducing agents paraquat and hydrogen peroxide. J Biol Chem. 1998; 273:22528-22536. 
23. Lei XG, Cheng WH, McClung JP. Metabolic regulation and function of glutathione peroxidase-1. Annu Rev Nutr. 2007; 27:41-61.

24. Fang W, Goldberg ML, Pohl NM, Bi X, Tong C, Xiong B, Koh TJ, Diamond AM, Yang W. Functional and physical interaction between the selenium-binding protein 1 (SBP1) and the glutathione peroxidase 1 selenoprotein. Carcinogenesis. 2010; 31:1360-1366.

25. Wang N, Chen Y, Yang X, Jiang Y. Selenium-binding protein 1 is associated with the degree of colorectal cancer differentiation and is regulated by histone modification. Oncol Rep. 2014; 31:2506-2514.

26. Forbes SA, Bindal N, Bamford S, Cole C, Kok CY, Beare D, Jia M, Shepherd R, Leung K, Menzies A, Teague JW, Campbell PJ, Stratton MR, et al. COSMIC: mining complete cancer genomes in the Catalogue of Somatic Mutations in Cancer. Nucleic Acids Res. 2011; 39:D945-950.

27. Forbes SA, Tang G, Bindal N, Bamford S, Dawson E, Cole C, Kok CY, Jia M, Ewing R, Menzies A, Teague JW, Stratton MR, Futreal PA. COSMIC (the Catalogue of Somatic Mutations in Cancer): a resource to investigate acquired mutations in human cancer. Nucleic Acids Res. 2010; 38:D652-657.

28. Kuphal S, Winklmeier A, Warnecke C, Bosserhoff AK. Constitutive HIF-1 activity in malignant melanoma. Eur J Cancer. 2010; 46:1159-1169.

29. Wang Y, Fang W, Huang Y, Hu F, Ying Q, Yang W, Xiong B. Reduction of selenium-binding protein 1 sensitizes cancer cells to selenite via elevating extracellular glutathione: a novel mechanism of cancer-specific cytotoxicity of selenite. Free Radic Biol Med. 2015; 79:186-196.

30. Scortegagna M, Martin RJ, Kladney RD, Neumann RG, Arbeit JM. Hypoxia-inducible factor-1alpha suppresses squamous carcinogenic progression and epithelialmesenchymal transition. Cancer Res. 2009; 69:2638-2646.

31. Li S, Yan T, Yang JQ, Oberley TD, Oberley LW. The role of cellular glutathione peroxidase redox regulation in the suppression of tumor cell growth by manganese superoxide dismutase. Cancer Res. 2000; 60:3927-3939.

32. Liu S, Qi Y, Ge Y, Duplessis T, Rowan BG, Ip C, Cheng H, Rennie PS, Horikawa I, Lustig AJ, Yu Q, Zhang H, Dong Y. Telomerase as an important target of androgen signaling blockade for prostate cancer treatment. Mol Cancer Ther. 2010; 9:2016-2025.

33. Franken NA, Rodermond HM, Stap J, Haveman J, van Bree C. Clonogenic assay of cells in vitro. Nat Protoc. 2006; 1:2315-2319.

34. Liao Y, Smyth GK, Shi W. feature Counts: an efficient general purpose program for assigning sequence reads to genomic features. Bioinformatics. 2014; 30:923-930.

35. Feinendegen LE, Pollycove M, Neumann RD. Low-dose cancer risk modeling must recognize up-regulation of protection. Dose Response. 2009; 8:227-252. 